\title{
HOXA4, down-regulated in lung cancer, inhibits the growth, motility and invasion of lung cancer cells
}

\author{
Shaofei Cheng ${ }^{1}$, Fengying Qian², Qin Huang ${ }^{3}$, Lirong Wei ${ }^{2}$, Yawen $\mathrm{Fu}^{2}$ and Yuzhen $\mathrm{Du}^{2}$
}

\begin{abstract}
The involvement of HOXA4 in colorectal cancer and epithelial ovarian cancer has been reported. Although it has been reported that the Hoxa4 gene is involved in the patterning of the mouse lung during embryonic development, little is known about the biological functions of HOXA4 in lung cancer. In the current study, HOXA4 expression was downregulated in lung cancer tissues when compared with non-cancerous tissues. HOXA4 expression was associated with tumor size, TNM stage, lymph node metastasis and prognosis. Bioinformatics analysis revealed that HOXA4 expression was negatively correlated with cell cycle, metastasis, and the Wnt signaling pathway. Moreover, HOXA4 overexpression in lung cancer cell lines suppressed cell proliferation, migration, and invasion. HOXA4 decreased the protein expression levels of $\beta$-catenin, Cyclin D1, c-Myc and Survivin, indicating the inhibition of Wht signaling. HOXA4 significantly increased the protein and mRNA levels of glycogen synthase kinase-3 3 (GSK3B) by promoting its transcription. Furthermore, inhibition of GSK3 $\beta$ by LiCl abolished the suppression of cell growth, migration, and invasion mediated by HOXA4. Overexpression of HOXA4 in xenograft tumors also decreased tumor growth and Wht signaling. Collectively, these data suggest that HOXA4 is a potential diagnostic and prognostic marker in lung cancer, and its overexpression could inhibit lung cancer progression in part by promoting GSK3 $\beta$ transcription.
\end{abstract}

\section{Introduction}

Lung cancer represents the leading cause of cancerrelated mortality in the world ${ }^{1}$. The most frequent type of lung cancer is non-small cell lung cancer (NSCLC), which accounts for $\sim 85 \%$ of lung cancer cases ${ }^{1}$. The overall survival for most patents with lung cancer is relatively low $^{2}$, mainly because of the lack of obvious initial symptoms and effective therapy. Recently, studies have identified several lung cancer-related pathways, including the epidermal growth factor receptor (EGFR) $)^{3,4}, \mathrm{p} 16^{\mathrm{INK} 4} /$ Cyclin $\mathrm{D} 1 / \mathrm{Rb}^{5}$ and Wnt signaling pathways ${ }^{6}$. Therapy targeting these pathways has provided a broad prospect for the treatment of lung cancer ${ }^{7,8}$.

\footnotetext{
Correspondence: Yuzhen Du (sd_duyz@sumhs.edu.cn)

${ }^{1}$ Department of Thoracic-cardiovascular Surgery, Shanghai Jiao Tong University

Affiliated Sixth People's Hospital, Shanghai, China

${ }^{2}$ Department of Laboratory Medicine, Shanghai Jiao Tong University Affiliated

Sixth People's Hospital, Shanghai, China

Full list of author information is available at the end of the article

Edited by G Dewson
}

HOXA4 belongs to the Homeobox (HOX) gene family, which is characterized by the presence of a 183-base pair DNA sequence (homeobox) that encodes a highly conserved homeodomain. HOX genes encode transcription factors that control cell differentiation and embryonic development by binding to the promoters of various target genes and regulating their expression ${ }^{9,10}$. Previous studies have investigated the regulation and expression of the Hoxa4 gene in mouse embryos ${ }^{11-13}$ and suggested that the Hoxa4 gene is involved in the patterning of the mouse lung ${ }^{14}$. Accumulated evidence has indicated the abnormal expression of development-associated genes in cancers and their contributions to carcinogenesis. HOXA4 is reportedly overexpressed in colorectal cancer ${ }^{15}$ and epithelial ovarian cancer ${ }^{16}$. Further study revealed that HOXA4 suppresses migration in ovarian cancer cell lines via $\beta 1$ integrin ${ }^{17}$. Although other members of the HOX gene family, such as HOXA5, HOXA10, HOXB3, HOXB4, and HOXC6 ${ }^{18,19}$, have been found to be

\section{(c) The Author(s) 2018}

(c) (i) Open Access This article is licensed under a Creative Commons Attribution 4.0 International License, which permits use, sharing, adaptation, distribution and reproduction cc) in any medium or format, as long as you give appropriate credit to the original author(s) and the source, provide a link to the Creative Commons license, and indicate if changes were made. The images or other third party material in this article are included in the article's Creative Commons license, unless indicated otherwise in a credit line to the material. If material is not included in the article's Creative Commons license and your intended use is not permitted by statutory regulation or exceeds the permitted use, you will need to obtain permission directly from the copyright holder. To view a copy of this license, visit http://creativecommons.org/licenses/by/4.0/. 

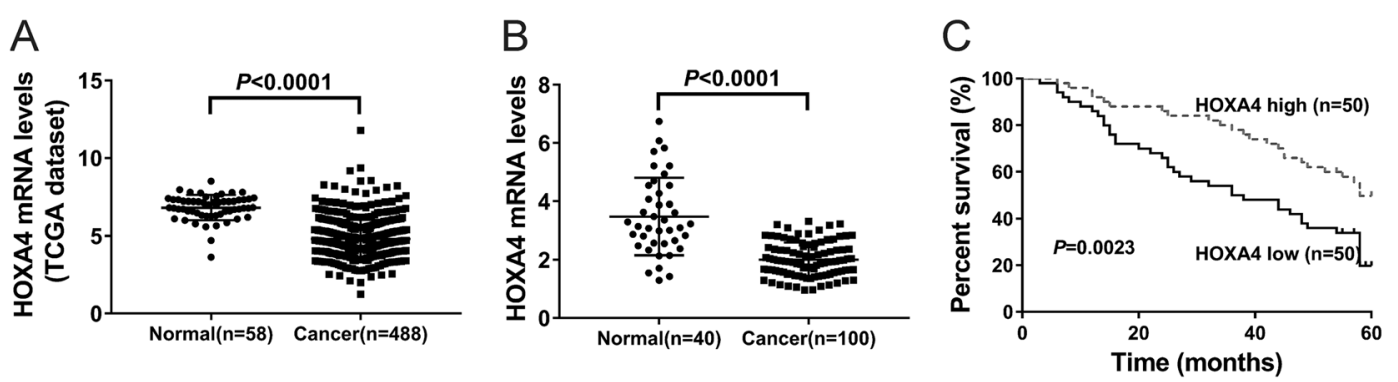

Fig. 1 Decreased expression of HOXA4 in human lung cancer tissues was associated with poor prognosis. a The expression levels of HOXA4 were analyzed in lung cancer and normal lung tissues from the TCGA lung cancer dataset. $\mathbf{b}$ HOXA4 mRNA expression was analyzed in lung cancer and normal lung tissues from our hospital. c Kaplan-Meier survival curve analysis of patients with high or low HOXA4 expression

overexpressed in lung cancer tissues compared with normal tissues, little is known about the expression and biological function of HOXA4 in lung cancer.

In this study, we demonstrated that HOXA4 was downregulated in lung cancer tissues compared with noncancerous tissues. We then performed functional characterization of HOXA4 in human lung cancer cell lines with HOXA4 overexpression or silencing. Our study showed that HOXA4 overexpression repressed the growth, motility and invasion of lung cancer cells and inhibited the Wnt pathway. Our findings suggest that HOXA4 may be a potential therapeutic target for lung cancer.

\section{Results}

HOXA4 expression is decreased in human lung cancer tissues

First, we analyzed HOXA4 expression in human lung cancer tissues by using a dataset downloaded from The Cancer Genome Atlas project (TCGA, https://tcga-data. nci.nih.gov/tcga/). Figure 1a shows that HOXA4 expression levels were decreased in lung cancer tissues $(n=488)$ compared with normal lung tissues $(n=58)(4.81 \pm 0.06$ vs. $6.82 \pm 0.10, P<0.0001)$. Further, quantitative reverse transcription-PCR (qRT-PCR) analysis was performed to examine the expression of HOXA4 in lung cancer samples and adjacent normal tissues obtained from patients at our hospital (Fig. 1b). We found that HOXA4 mRNA expression was lower in tumor tissues $(n=100)$ than in normal lung tissues $(n=40)(2.01 \pm 0.06$ vs. $3.49 \pm 0.21, P$ $<0.0001)$. These data suggest the clinical significance of HOXA4 in lung cancer diagnosis.

Decreased expression of HOXA4 is associated with tumor size, TNM stage, lymph node metastasis and poor prognosis in lung cancer

We then examined the correlation between HOXA4 expression and clinical pathological features. The 100 lung cancer patients were split into two groups: the HOXA4 high expression group and HOXA4 low
Table 1 Correlation of HOXA4 expression with patients' features

\begin{tabular}{lll}
\hline Variables $\quad$ All cases & HOXA4 mRNA & $P$ value \\
\cline { 2 - 3 } & $\operatorname{Low}(n=50) \quad H i g h(n=50)$ & \\
\hline
\end{tabular}

\begin{tabular}{lllll}
\hline $\begin{array}{l}\text { Age at surgery } \\
<55\end{array}$ & 41 & 22 & 19 & 0.6845 \\
$\geq 55$ & 59 & 28 & 31 & \\
Gender & & & & 0.4230 \\
Male & 53 & 24 & 29 & \\
Female & 47 & 26 & 21 & 0.5269 \\
Smoking status & & & & \\
Smoker & 34 & 19 & 15 & $0.0262^{*}$ \\
Non-smoker & 66 & 31 & 35 & \\
Tumor size & & & & \\
$<5$ cm & 44 & 16 & 28 & $0.0088^{* *}$ \\
$\geq 5$ cm & 56 & 34 & 22 & \\
TNM stage & & & 30 &
\end{tabular}

Lymphnode metastasis

\begin{tabular}{lllll} 
Absent & 60 & 23 & 37 & $0.0076^{* *}$ \\
Present & 40 & 27 & 13 & \\
\hline
\end{tabular}

Fisher's exact test; ${ }^{*} P<0.05,{ }^{* *} P<0.01$.

expression group. As analyzed by Fisher's exact test (Table 1), we found that HOXA4 expression in lung cancer was significantly associated with tumor size $(P=$ 0.0262), TNM stage $(P=0.0088)$ and lymph node metastasis $(P=0.0076)$. However, HOXA4 expression was not correlated with age $(P=0.6845)$, gender $(P=$ $0.4230)$, or smoking status $(P=0.5269)$ in lung cancer.

Kaplan-Meier analysis and the log-rank test were then conducted to assess the relationship between HOXA4 


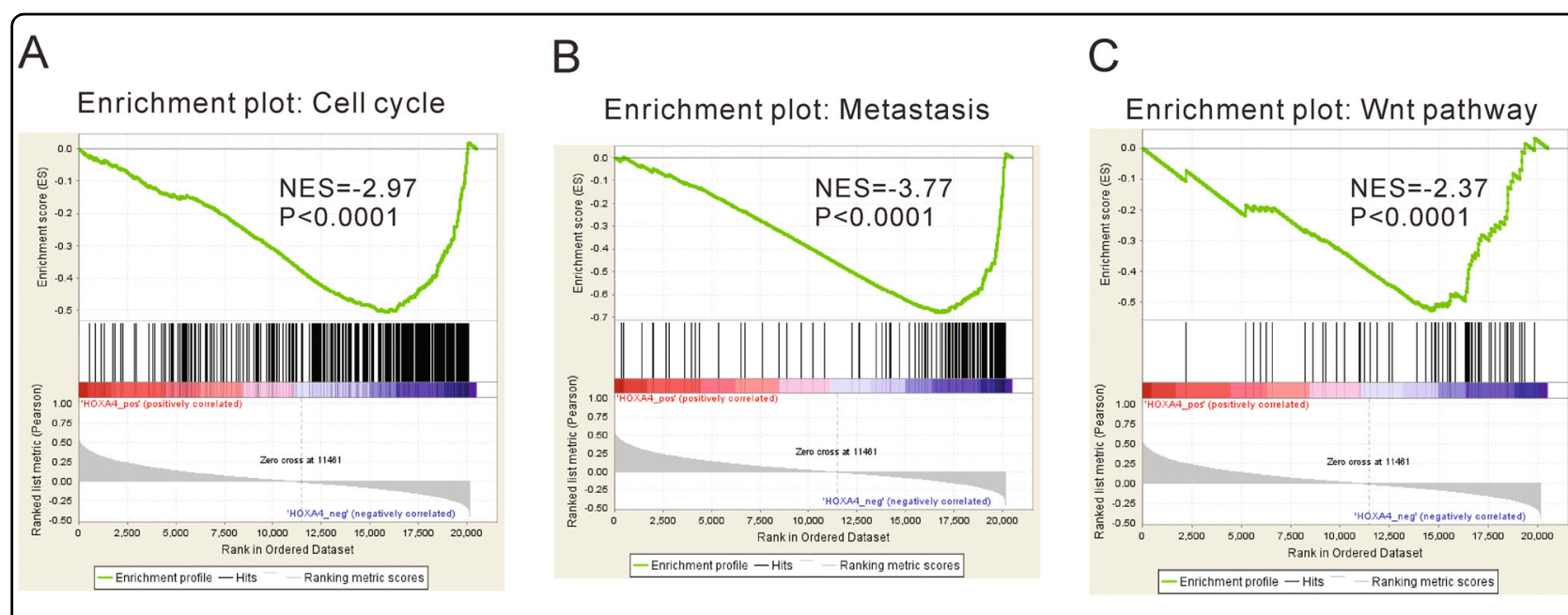

Fig. 2 GSEA analysis showed that HOXA4 was negatively associated with cell cycle (a), metastasis (b), and the Wnt signaling pathway (c) in the TCGA lung cancer samples

expression and the outcomes of lung cancer patients after surgery. The overall survival (OS) curves (Fig. 1c) showed that patients with lower HOXA4 expression levels had poorer OS $(P<0.01)$. These results suggest that HOXA4 expression may be a novel prognostic marker for lung cancer.

\section{Analysis of HOXA4-associated pathways}

Gene set enrichment analysis (GSEA) was performed to evaluate pathways that were associated with HOXA4 expression in the TCGA lung cancer samples. The results revealed that HOXA4 expression was negatively correlated with cell cycle, metastasis and the Wnt signaling pathway (Fig. 2), which implied that HOXA4 may affect the growth, invasion and migration of lung cancer.

\section{Manipulation of HOXA4 expression in lung cancer cell lines}

To investigate the biological implications of HOXA4 in lung cancer, we first analyzed the mRNA and protein expression levels of HOXA4 among 5 lung cancer cell lines and 1 normal lung epithelium cell line via qRT-PCR and western blot. The expression of HOXA4 was significantly down-regulated in lung cancer cell lines, especially in the NCI-H1975 and NCI-H446 cell lines (Fig. 3a). We then used lentiviral transduction to overexpress HOXA4 in NCI-H1975 and NCI-H446 cells and knocked down HOXA4 expression in NCI-1299 cells, which had relatively high expression of HOXA4. qRT-PCR and western blot analyses were conducted at $48 \mathrm{~h}$ after transduction. As illustrated in Fig. 2b, c, vector or control shRNA (shNC) viral transduction had no effects on HOXA4 expression. The mRNA and protein levels of HOXA4 were significantly increased in cells transfected with HOXA4 virus compared with wild-type cells (WT) or cells transfected with vector virus (Fig. 3b). Three different shRNAs (shHOXA4-1, 2, and 3) remarkably reduced the levels of HOXA4 in NCI-1299 cells among which shHOXA4-3 (referred to as shHOXA4) had the best knockdown efficiency (Fig. 3c) and was selected for the following functional assays.

\section{HOXA4 inhibits growth and promotes apoptosis of lung cancer cells in vitro}

Cell Count Kit-8 (CCK-8) assays were carried out to explore the functions of HOXA4 regarding lung cancer cell proliferation. Ectopic expression of HOXA4 significantly inhibited cell growth in NCI-H1975 and NCI-H446 cells compared with the controls (WT and vector) (Fig. 4a). In contrast, knocking down HOXA4 expression promoted cell proliferation in NCI-H1299 cells (Fig. 4b). Annexin V staining followed by cytometry analysis was performed to explore the effects of HOXA4 on lung cancer cell apoptosis. HOXA4 overexpression significantly promoted cell apoptosis in NCI-H1975 and NCI-H446 cells compared with controls (WT and vector) (Fig. 4c), whereas knocking down HOXA4 expression had the opposite effect on NCI-H1299 cells (Fig. 4d). These data imply that HOXA4 may act as a tumor suppressor involved in the inhibition of cancer cell proliferation and promotion of cancer cell apoptosis.

\section{HOXA4 suppresses lung cancer cell migration and invasion}

Transwell assays with or without Matrigel were performed to determine the influence of HOXA4 on lung cancer cell invasion and migration, respectively. Compared with the controls, the ectopic expression of HOXA4 in NCI-H1975 and NCI-H446 cells led to a notable decrease in cell migration and invasion (Fig. 5a, b). However, knocking down HOXA4 expression in NCIH1299 cells promoted cell migration and invasion 


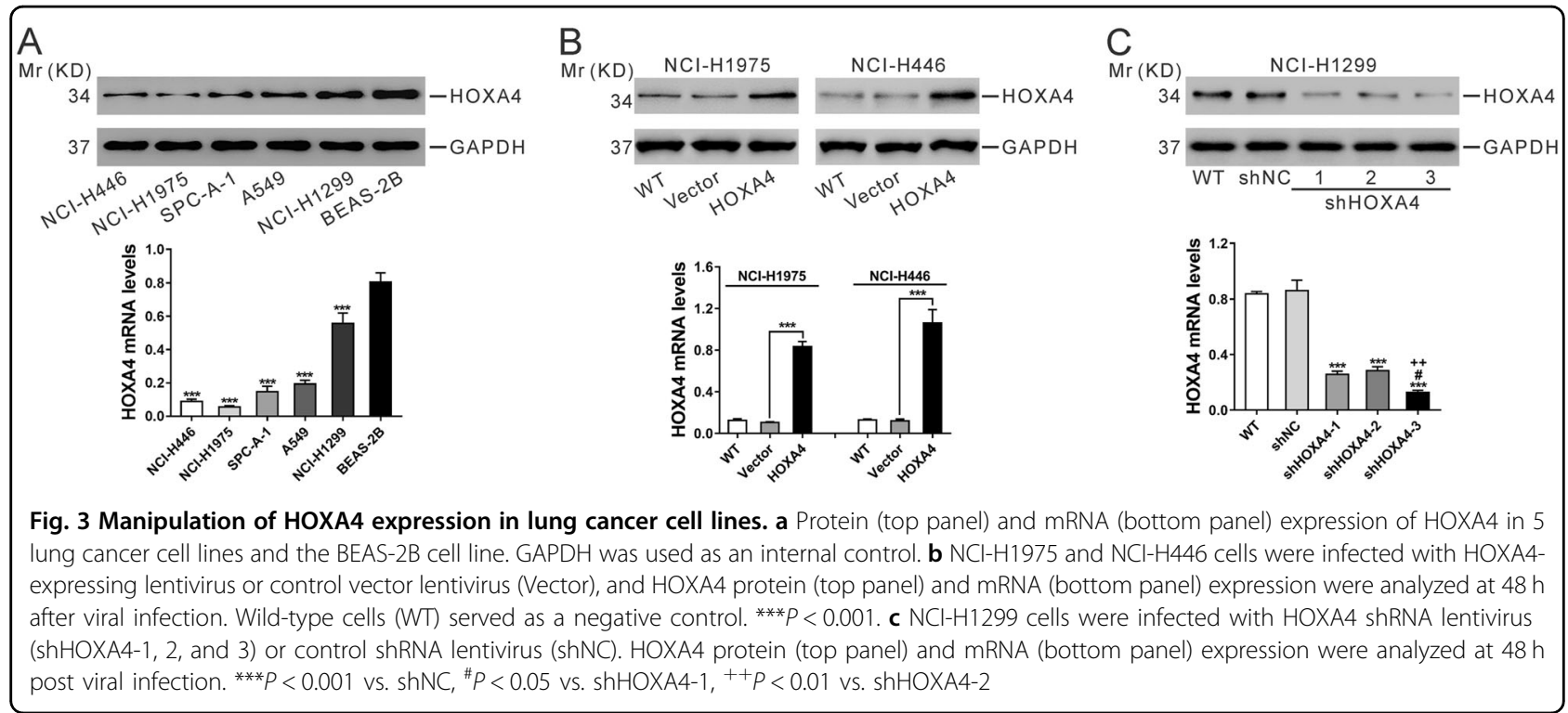

(Fig. 5c). These data indicate that HOXA4 suppresses lung cancer cell migration and invasion.

\section{HOXA4 suppresses the Wnt signaling pathway in lung cancer cells}

Activation of the Wnt pathway can induce cell proliferation and invasion and is likely to be crucial in promoting carcinogenesis ${ }^{20}$. Considering that HOXA4 expression was negatively correlated with the Wnt signaling pathway (Fig. 2c), we supposed that HOXA4 could suppress Wnt signaling. Expression of the major component in the Wnt pathway (glycogen synthase kinase-3 $\beta$ [GSK3 3$]$ ) and downstream effectors of the Wnt pathway ( $\beta$-catenin, Cyclin D1, c-Myc, and Survivin) was assessed by western blot in lung cancer cells in which HOXA4 had been overexpressed or knocked down. As shown in Fig. 6a, the ectopic expression of HOXA4 in NCI-H1975 and NCI-H446 cells resulted in a notable increase in GSK3 $\beta$ protein levels and an obvious decrease in $\beta$-catenin, Cyclin D1, c-Myc, and Survivin protein levels. Knocking down HOXA4 expression in NCI-H1299 cells showed the opposite effect.

Similarly, qRT-PCR results revealed that the mRNA level of GSK3 $\beta$ was significantly increased following the overexpression of HOXA4 in NCI-H1975 and NCI-H446 cells but markedly decreased in NCI-H1299 cells in which HOXA4 was silenced (Fig. 6c, d).

Further, GSK3 $\beta$ promoter-luciferase assays were conducted in NCI-H1975 and NCI-H446 cells in which HOXA4 had been overexpressed or silenced. The results showed that the relative luciferase activity was markedly enhanced by HOXA4 overexpression but notably reduced by HOXA4 knockdown (Fig. 6e). The results of a chromatin immunoprecipitation (ChIP)-quantitative PCR (qPCR) assay showed that the binding level of HOXA4 at the GSK3 $\beta$ promoter was consistent with the expression level of HOXA4 (Fig. 6f). These data confirmed that HOXA4 could bind to the GSK3 $\beta$ promoter and that the HOXA4 expression level affected GSK3 $\beta$ promoter activity and transcription.

\section{The Wnt signaling pathway mediates HOXA4 effects on cell growth, migration and invasion}

To clarify whether the Wnt signaling pathway mediates the effects of HOXA4 on cell growth, migration and invasion, NCI-H1975 cells with HOXA4 overexpression were exposed to $\mathrm{LiCl}$, which activates Wnt signaling $^{21}$ (Fig. 7). HOXA4 overexpression markedly suppressed cell growth, migration and invasion, and this effect was abolished by $\mathrm{LiCl}$ stimulation. These data confirmed that the Wnt signaling pathway is involved in the functions of HOXA4 on cell growth, migration and invasion.

HOXA4 inhibits lung cancer cell tumorigenesis in vivo

To determine whether HOXA4 expression could affect tumorigenesis, NCI-H1975 cells transduced with a HOXA4 overexpression virus or vector virus were transplanted into nude mice. We found that the tumor growth in the HOXA4 overexpression group was significantly slower than that in the vector group (Fig. 8a). At 33 days after inoculation, tumor size (Fig. 8b), and weight (Fig. 8c) was noticeably decreased in the HOXA4 overexpression group compared with the vector group. Moreover, Ki67positive signals from the xenograft tumors also declined in the HOXA4 overexpression group (Fig. 8d). Western 


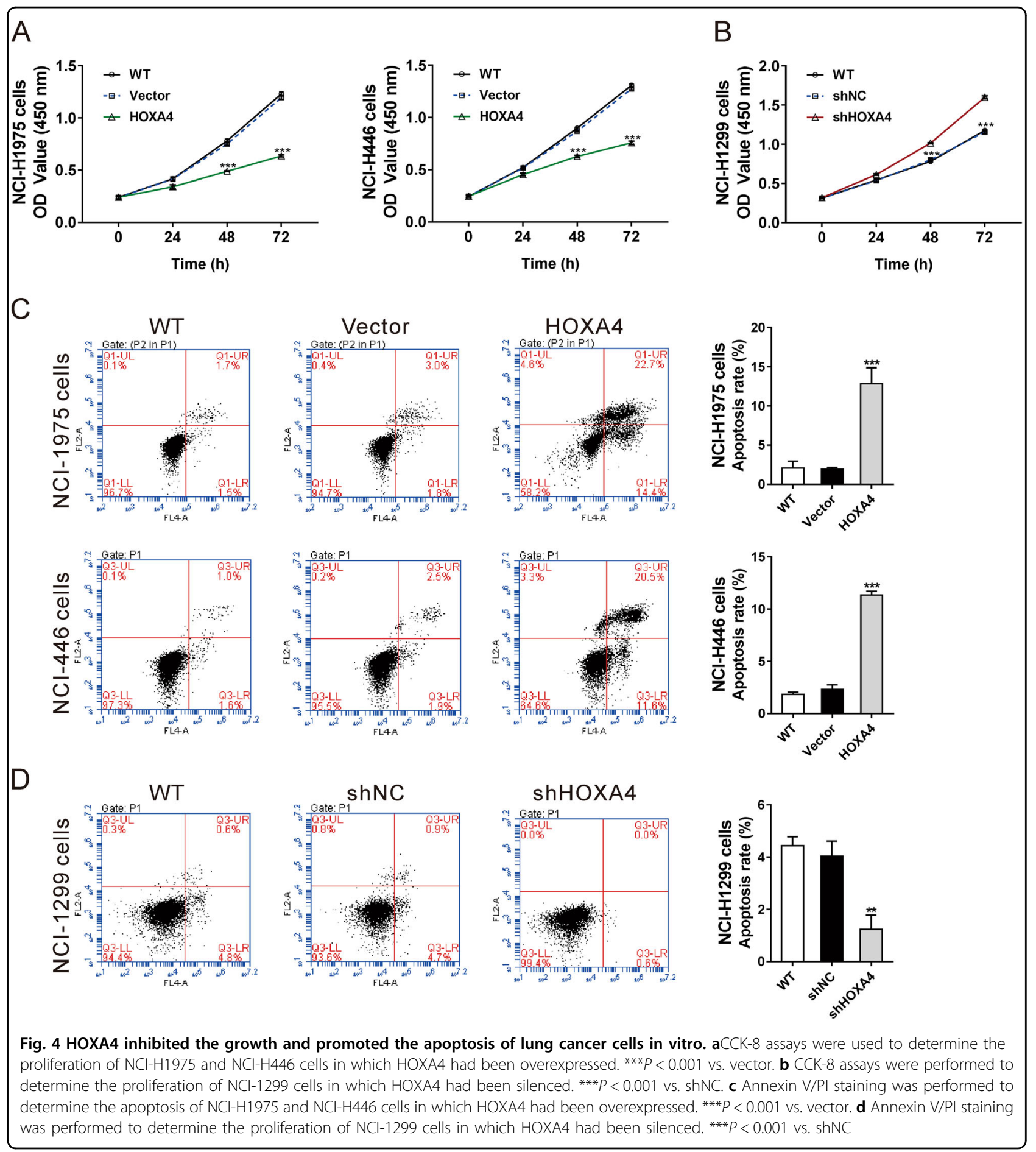

blotting analysis revealed that tumor tissues formed from HOXA4-overexpressing cells displayed higher levels of HOXA4 and GSK3 $\beta$ and lower levels of $\beta$-catenin than did cells in the vector group. These results suggest that HOXA4 overexpression significantly reduces the proliferation capacity of lung cancer cells and suppresses the Wnt signaling pathway in vivo.

\section{Discussion}

HOXA4 is reportedly overexpressed in colorectal cancer $^{15}$ and epithelial ovarian cancer ${ }^{16}$. The Hoxa4 gene is involved in the patterning of the mouse lung during embryonic development ${ }^{14}$. We hypothesized that HOXA4 may be associated with lung carcinogenesis. To test this hypothesis, we analyzed the expression of HOXA4 in the 

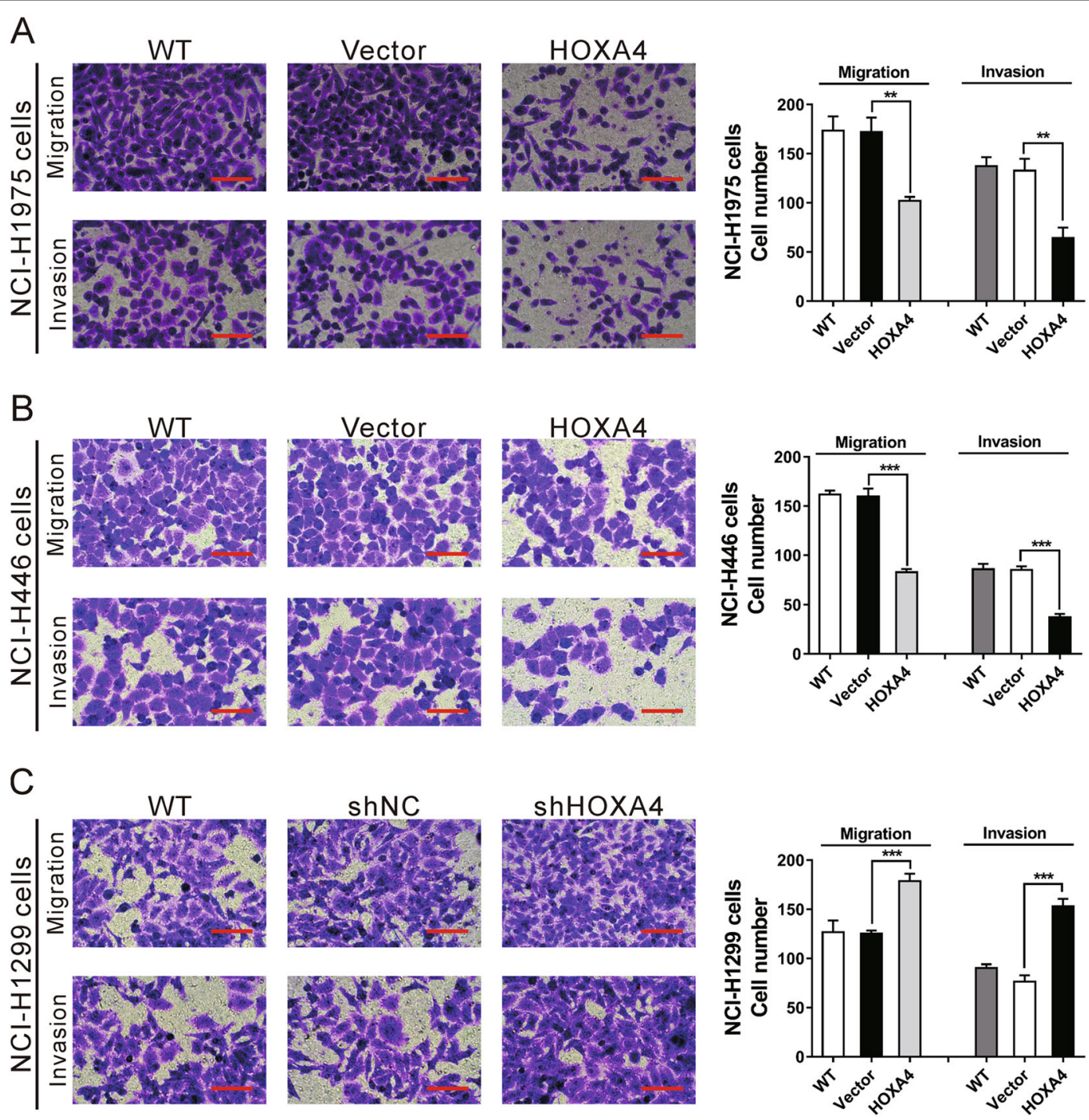

Fig. 5 HOXA4 suppressed lung cancer cell migration and invasion. Transwell assays with or without Matrigel were performed to determine the invasion and migration abilities of $\mathrm{NCl}-\mathrm{H} 1975$ (a) and NCl-H446 (b) cells with HOXA4 overexpression and NCl-1299 cells (c) with HOXA4 silencing. Magnification: $\times 200$. Scale bars $=100 \mu \mathrm{m} .{ }^{* * *} \mathrm{P}<0.001$

TCGA lung cancer dataset and our own patient cohort. We found that HOXA4 levels were significantly lower in lung cancer tissues compared with normal lung tissues (Fig. 1). We also observed that HOXA4 expression in lung cancer was significantly associated with tumor size, TNM stage, lymph node metastasis and overall survival (Fig. 2 and Table 1). These findings indicated that HOXA4 can be used as a potential diagnostic and prognostic marker for lung cancer.

The functions of HOXA4 in cancer progression have been rarely studied except for its role in suppressing migration in ovarian cancer cell lines ${ }^{17}$. In the present study, we explored the effects of HOXA4 expression levels on the growth, migration and invasion of lung cancer cells by manipulating HOXA4 expression with lentiviral transduction (Figs. 4, 5, and 8). To our knowledge, this is the first report that HOXA4 may potentially serve as a tumor suppressor in lung cancer. We also showed that overexpression of HOXA4 significantly promoted cell apoptosis (Fig. 4c, d), suggesting that increased cell apoptosis is one of the potential reasons for the decreased proliferation observed in HOXA4-overexpressing cells.

The Wnt signaling pathway plays an important role in lung cancer tumorigenesis and prognosis ${ }^{22}$. Prior studies have suggested that HOXA5 represses the Wnt signaling activity in colon cancer cell lines ${ }^{23}$, whereas HOXA9 and HOXA10 activate Wnt signaling activity in human CD34 + umbilical cord blood cells ${ }^{24}$. However, it is not known whether HOXA4 can affect the Wnt signaling pathway. Here, GSEA on the TCGA dataset showed that HOXA4 


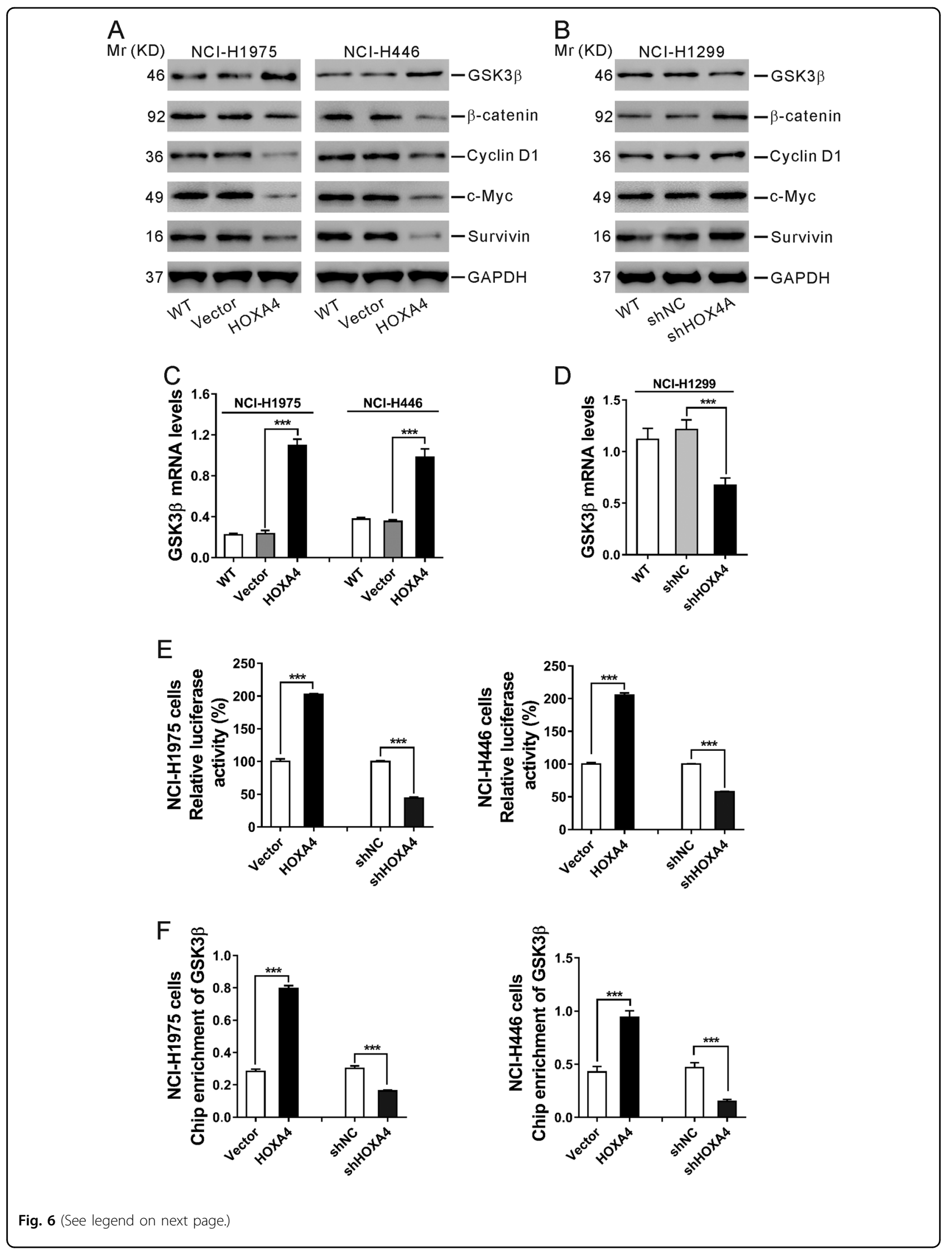


(see figure on previous page)

Fig. 6 HOXA4 suppressed the Wnt signaling pathway in lung cancer cells. a, b Western blot analysis of GSK3 $\beta, \beta-c a t e n i n, C y c l i n ~ D 1, c-M y c$ and Survivin in NCl-H1975 and NCl-H446 cells with HOXA4 overexpression (a) and NCl-H1299 cells with HOXA4 silencing (b). GAPDH was used as a loading control. c, d qRT-PCR analysis of GSK3 $\beta$ mRNA levels in NCl-H1975 and NCl-H446 cells with HOXA4 overexpression (c) and NCl-H1299 cells with HOXA4 silencing (d). e A luciferase reporter assay was performed to evaluate GSK3 $\beta$ promoter activity in NCl-H1975 and NCI-H446 cells with HOXA4 overexpression or silencing. $\mathbf{f}$ ChIP-qPCR for the GSK3ß promoter in NCI-H1975 and NCl-H446 cells with HOXA4 overexpression or silencing. ***P $<0.001$

expression was negatively correlated with the Wnt signaling pathway (Fig. 2c). Ectopic expression of HOXA4 led to an obvious decrease in the protein levels of downstream effectors of the Wnt pathway ( $\beta$-catenin, Cyclin D1, c-Myc, and Survivin) (Fig. 6a), which are involved in the development of lung cancer. Reduced $\beta$ catenin expression is a poor prognostic factor for patients with lung cancer ${ }^{25}$. Genetic alteration of Cyclin D1 is a prognostic factor in NSCLC ${ }^{26,27}$. Suppression of c-Myc, a well-known oncogene, decreases proliferation in lung cancer cell lines ${ }^{28}$. Inhibition of Survivin expression increases apoptosis in NSCLC cells ${ }^{29}$. Our findings suggest that Wnt signaling may be involved in HOXA4 suppression-mediated tumorigenesis, although we could not exclude other factors. Further studies are needed to clarify this issue using specific knockdown of the proposed targets ( $\beta$-catenin, Cyclin D1, c-Myc, and Survivin). GSK3 $\beta$, a major component in the Wnt pathway, promotes the phosphorylation and degradation of $\beta$-catenin, thus inhibiting Wnt signaling ${ }^{22}$. Dysregulation of GSK3 $\beta$ has been observed in various human cancers. GSK3 $\beta$ may functions as a tumor suppressor ${ }^{30-36}$ and tumor promoter $^{37-41}$, depending on the cell types and signaling pathways involved. For example, activation of GSK3 $\beta$ induces apoptosis, accumulation of $\beta$-catenin and suppression of Cyclin D1 expression in human breast cancer cells $^{33}$. GSK3 $\beta$ has been proposed to be involved in breast cancer cell metastasis via regulation of Snail1 activity ${ }^{36}$. The overexpression of inactivated GSK3 $\beta$ may be a worse prognostic factor for patients with lung cancer $^{34}$. In contrast, GSK3 $\beta$ has been reported to be upregulated in NSCLC tissues and to positively regulate tumor cell proliferation $^{38}$. Here, we found that HOXA4 overexpression increased GSK3 $\beta$ protein and mRNA levels. The relative luciferase activity of GSK3 $\beta$ and enrichment of the GSK3 $\beta$ promoter was markedly enhanced by HOXA4 overexpression but notably reduced by HOXA4 knockdown (Fig. 6). Accordingly, we speculated that HOXA4 might increase GSK3 $\beta$ expression via promoting its transcription and then inhibiting Wnt signaling. Moreover, $\mathrm{LiCl}$ treatment, which can inactivate GSK3 $\beta$, markedly promoted the cell growth and invasion of HOXA4-overexpressing cells (Fig. 7). Our data indicated that inactivation of GSK3 $\beta$ may promote tumorigenesis, which was consistent with a previous study ${ }^{34}$. Our data also indicated that the Wnt signaling pathway mediates the functions of HOXA4 regarding cell growth, migration and invasion.

In summary, down-regulation of HOXA4 was associated with poor prognosis of lung cancer. The ectopic expression of HOXA4 in lung cancer cells decreased cell proliferation, migration and invasion as well as Wnt signaling. HOXA4 is a potential tumor suppressor in lung cancer.

\section{Materials and methods \\ Patients and tissue samples}

This study was approved by the Research Ethics Committee of Shanghai Jiao Tong University Affiliated Sixth People's Hospital. A total of 100 patients with lung cancer who underwent surgical resection at the Department of Thoracic-cardiovascular Surgery, Shanghai Jiao Tong University Affiliated Sixth People's Hospital, were enrolled in this study. Written informed consent was obtained from all enrolled patients. Clinical information was obtained by review of medical records. The follow-up period lasted 5 years. Primary lung cancer tissues $(n=$ $100)$ and adjacent non-cancerous tissues $(n=40)$ were obtained during surgery, immediately frozen in liquid nitrogen and stored at $-80^{\circ} \mathrm{C}$ until use.

\section{Quantitative reverse transcription-PCR (qRT-PCR)}

Total RNA was isolated from cells or tissues using TRIzol reagent (Life Science, Carlsbad, CA, USA) per the manufacturer's instructions. Total RNA was treated with DNase and then reverse transcribed to cDNA using the RevertAid First-Strand cDNA Synthesis Kit (Thermo Scientific, Rockford, IL, USA). qRT-PCR analysis was performed with SYBR Green qPCR Master Mixes (Thermo Scientific) on an ABI 7300 system (Applied Biosystem, Foster City, CA, USA). The messenger RNA (mRNA) levels of HOXA4, glycogen synthase kinase-3 $\beta$ (GSK3 $\beta$ ) and GAPDH (internal control) were quantified using the following primers: HOXA4, forward: 5'ATAACGGAGGGGAGCCTAAG- $3^{\prime}$, reverse: $5^{\prime}$ GCTCAGACAAACAGAGCGTG-3'; GSK3 $\beta$, forward: 5' AAGGTTAGCTGGTAACTGTAGG -3', reverse: 5'TTTCTGGGTACTGGTTCACTTC - $3^{\prime}$; GAPDH, forward: 5'-CACCCACTCCTCCACCTTTG-3', reverse: $5^{\prime}$ CCACCACCCTGTTGCTGTAG-3'. All samples were 


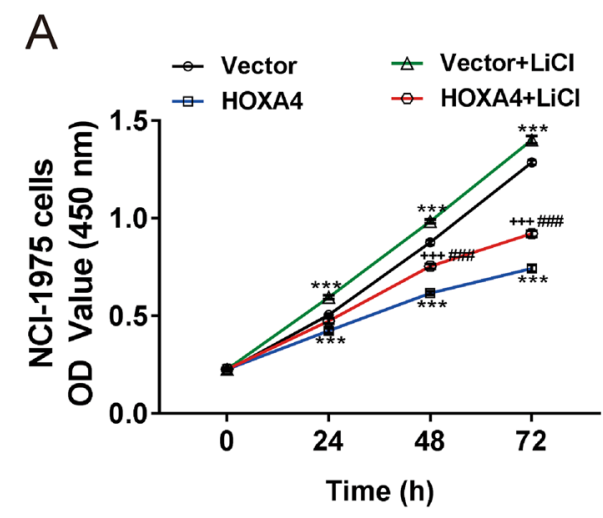

B
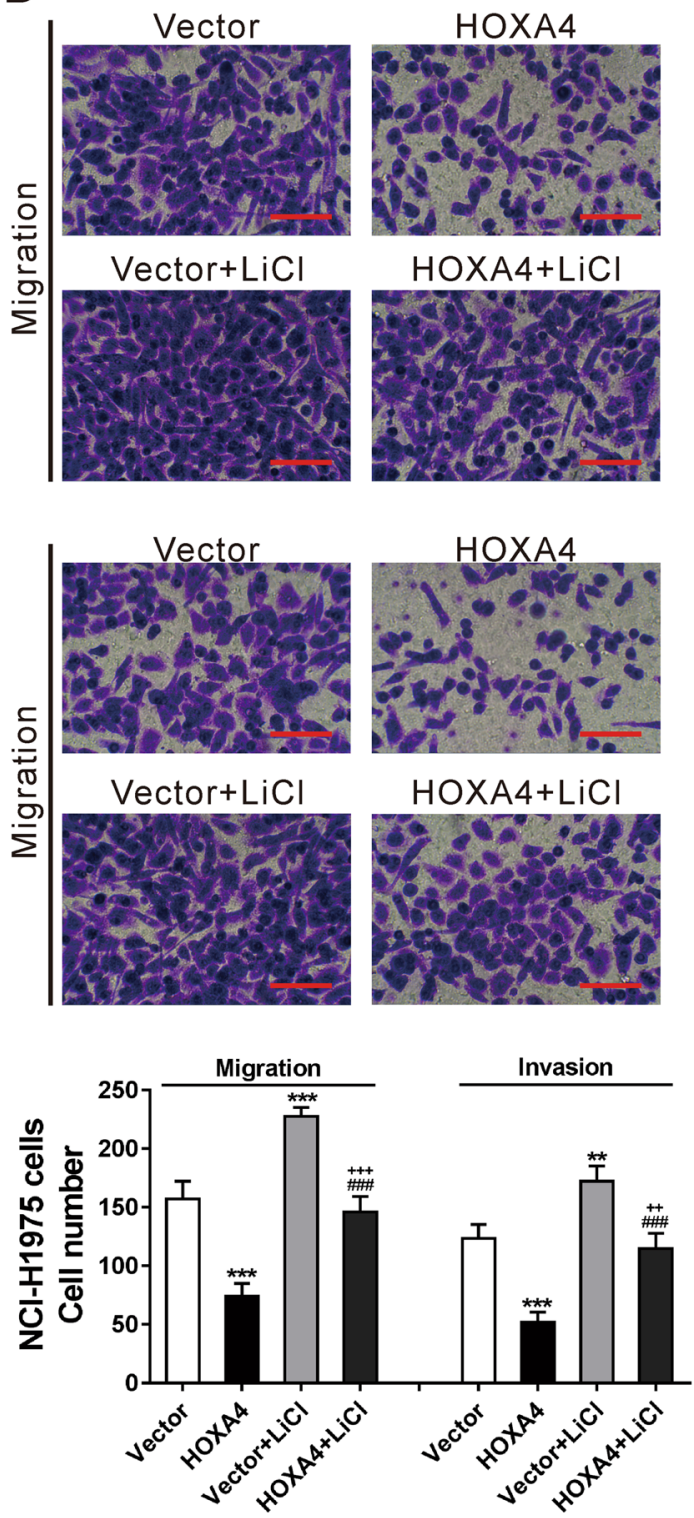

Fig. 7 The Wnt signaling pathway mediated the effects of HOXA4 on cell growth and invasion. $\mathrm{NCl}-\mathrm{H} 1975$ cells were infected with HOXA4 overexpression lentivirus or control vector lentivirus (Vector). $\mathrm{LiCl}(20 \mathrm{mM})$ was added at $24 \mathrm{~h}$ post viral infection. CCK-8 (a) and transwell assays (b) were performed to determine the proliferation, invasion and migration abilities of $\mathrm{NCl}-\mathrm{H} 1975$ cells with $\mathrm{LiCl}$ treatment and HOXA4 overexpression. ${ }^{* *} P<0.001$ vs. Vector; ${ }^{\# \# \#} P<0.001$ vs. HOXA4; ${ }^{++} P<0.01$ and ${ }^{+++} P<0.001$ vs. Vector $+\mathrm{LiCl}$

run in triplicate. The mRNA levels of target genes were normalized to GAPDH mRNA using the $2^{-\Delta \Delta C t}$ $\operatorname{method}^{42}$.

\section{Bioinformatics analysis}

The lung cancer dataset, which included 488 lung cancer tissues and 58 normal lung tissues, was obtained from The Cancer Genome Atlas project (TCGA, https:// tcga-data.nci.nih.gov/tcga/). Student's $t$-test was then used to compare HOXA4 expression levels between lung cancer and normal lung tissues.

Gene set enrichment analysis (GSEA) was performed to identify pathways associated with HOXA4 mRNA expression levels in the TCGA lung cancer dataset, as previously described ${ }^{43}$. GSEA software was obtained from the Broad Institute (http://www.broad.mit.edu/gsea).

\section{Cell lines}

NCI-H446, NCI-H1975, SPC-A-1, A549, NCI-H1299, BEAS-2B, and 293T cells were obtained from the Cell Bank of the Shanghai Biology Institute (Shanghai, China) and maintained in a $5 \% \mathrm{CO}_{2}$ incubator at $37^{\circ} \mathrm{C}$. A549 and $293 \mathrm{~T}$ cells were grown in DMEM, and other cells were cultured in RPMI-1640. All media were supplemented with $10 \%$ fetal bovine serum (FBS) and 1\% penicillin/ streptomycin (Life Technologies).

\section{Lentiviral production and infection}

DNA oligonucleotides encoding three small hairpin RNAs (shRNAs) against HOXA4 (target sequences: shHOXA4-1, 5' - CCAACTACATCGAGCCCAA-3', shHOXA4-2, 5'-TCCATGTCAGCGCCGTTAA-3', shHOXA4-3, 5'-AGATGCGATCCTCCAATTC-3'), or a negative control (shNC) were inserted into the lentiviral vector pLKO.1-puro (Addgene, Cambridge, MA, USA). Human HOXA4 cDNA (synthesized by Genewiz, Suzhou, China) was inserted into the EcoRI and BamHI sites of the lentiviral vector pLVX-puro (Clontech, Palo Alto, CA, USA). For lentiviral production, 293T cells were transfected with the lentiviral vector along with packaging plasmids using Lipofectamine 2000 (Life Science) according to the manufacturer's instructions. At $48 \mathrm{~h}$ and $72 \mathrm{~h}$ after 


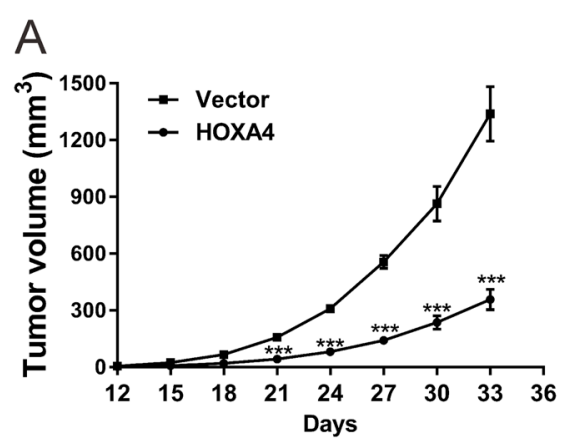

B
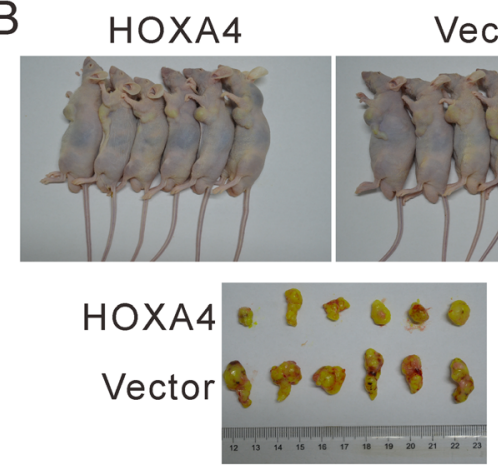

C
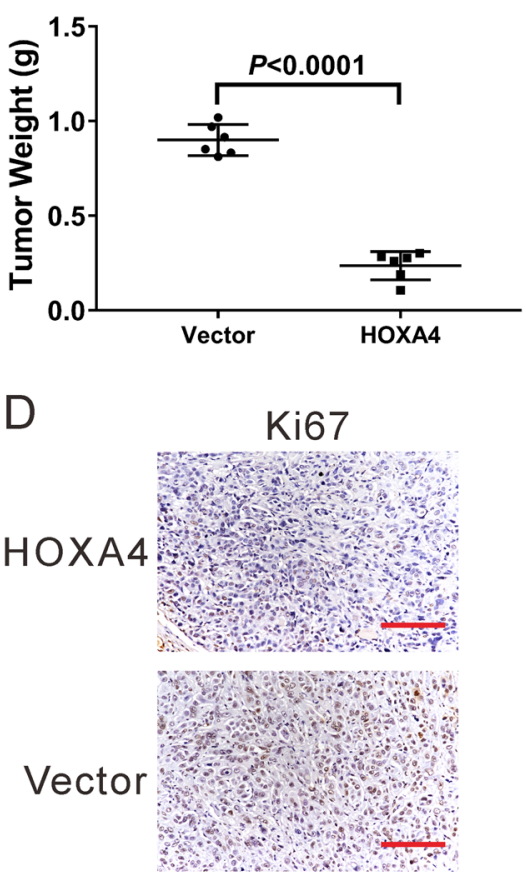

E

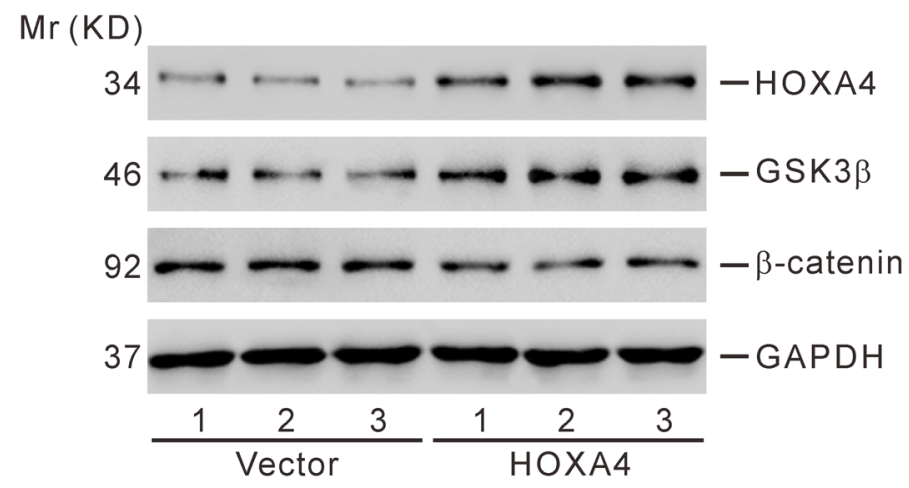

Fig. 8 HOXA4 inhibited tumorigenesis of lung cancer cells in vivo. NCl-H1975 cells transduced with empty vector or HOXA4-Overexpressing virus were injected into nude mice $(n=6)$. a Tumor volumes were measured every 3 days after injection. ${ }^{* * *} P<0.001$. b, c At 33 days after injection, xenograft tumors were recovered and weighed. $\mathbf{d}$ The tumor sections were stained for IHC using antibodies against Ki67. Magnification: $\times 200$. Scale bars $=100 \mu \mathrm{m}$. e Western blotting was performed to assess the protein levels of HOXA4, GSK3 $\beta$, and $\beta$-catenin in xenograft tumors. Data representing three replications are shown

transfection, culture media was collected, pooled and filtered. Lung cancer cell lines were infected with the indicated lentivirus, and HOXA4 expression was determined by qRT-PCR and western blotting at $48 \mathrm{~h}$ after infection.

\section{Western blotting}

An antibody against HOXA4 from Abcam and antiGSK3 $\beta$, anti- $\beta$-catenin, anti-Cyclin D1, anti-c-Myc, antiSurvivin and anti-GAPDH antibodies from Cell Signaling Technology were used in western blotting analysis. Cells or tissues were lysed in RIPA buffer (Solarbio, Beijing, China) with a protease inhibitor cocktail (Sigma Aldrich, St. Louis, MO, USA). Protein concentration was evaluated with a BCA (bicinchoninic acid) protein assay kit (Thermo Scientific). Approximately $30 \mu \mathrm{g}$ protein lysate of each sample was separated by $10 \%$ or $15 \%$ SDS-PAGE and transferred onto nitrocellulose membranes (Millipore, Bedford, MA, USA). The membranes were incubated with primary antibodies at $4{ }^{\circ} \mathrm{C}$ overnight and then with a horseradish peroxidase (HRP)-conjugated secondary antibody at room temperature for $1 \mathrm{~h}$. The target 
proteins were detected with the enhanced chemiluminescence substrate (Millipore).

\section{Cell growth assay}

To compare cell growth rates, $3 \times 10^{3}$ cells in $100 \mu \mathrm{l}$ media were seeded in 96-well plates and cultured overnight. The cells were transduced with the desired virus, and cell growth was assessed at $0,24,48$, and $72 \mathrm{~h}$ after viral transduction using the Cell Count Kit-8 (CCK-8) assay (SAB biotech., College Park, MD, USA) according to the manufacturer's instructions. Briefly, the culture media was replaced with $10 \%$ CCK- 8 solution (in culture medium) and incubated at $37^{\circ} \mathrm{C}$ for $1 \mathrm{~h}$. Optical density (OD) at $450 \mathrm{~nm}$ was detected with a microplate reader (BioTek, Winooski, VT, USA). Experiments were performed in triplicate and repeated three times.

\section{Cell apoptosis assay}

To compare cell apoptosis rates, $5 \times 10^{5}$ cells were seeded in 6-well plates and cultured overnight. The cells were transduced with the desired virus, and cell apoptosis was assessed at $48 \mathrm{~h}$ after viral transduction using the Annexin V Apoptosis Detection Kit (eBioscience, San Diego, CA, USA) according to the manufacturer's instructions. Briefly, the cells were harvested and washed in PBS. The cells were then incubated with Annexin V for $15 \mathrm{~min}$ followed by propidium iodide (PI) for $5 \mathrm{~min}$ in the dark. Cell apoptosis was analyzed in a flow cytometer (BD Biosciences, Franklin Lakes, NJ, USA). Experiments were performed in triplicate and repeated three times.

\section{Transwell assay}

Cellular migration and invasion were determined by Transwell assay. For the migration assay, cells grown in 6well plates were transduced with the desired virus. After $24 \mathrm{~h}$, the cells were serum starved overnight. The cells were then trypsinized, $1 \times 10^{4}$ cells in $300 \mu \mathrm{l}$ serum-free medium were added to the upper chamber, and $700 \mu \mathrm{l}$ media containing 10\% FBS was added to the lower chamber. Following growth at $37^{\circ} \mathrm{C}$ for $24 \mathrm{~h}$, the migrating cells were fixed in $4 \%$ paraformaldehyde and stained with $0.5 \%$ crystal violet. The stained cells were then counted under a microscope. Experiments were performed in triplicate.

The invasion assay was performed in the same manner as the migration assay except that the upper chamber was coated with $1 \mathrm{mg} / \mathrm{ml}$ Matrigel (BD Biosciences) prior to the experiments.

\section{Luciferase assay}

The human GSK3 $\beta$ promoter ${ }^{44}$ was amplified from genomic DNA and cloned into the KpnI and BglII sites of pGL3-basic vector (Promega, Madison, WI, USA). NCIH1975 and NCI-H446 cells were transfected with pRL-TK
(Promega) and the pGL3-GSK3 $\beta$ promoter using lipofectamine 2000 reagent, infected with HOXA4-expressing or vector lentivirus, or infected with HOXA4 shRNA (shHOXA4) or control shRNA (shNC). At $48 \mathrm{~h}$ following transfection, cells were lysed, and luciferase activity was determined using a dual-luciferase reporter assay according to the manufacturer's protocol (Promega). Values were normalized to that of Renilla luciferase and then to a control.

\section{Chromatin immunoprecipitation (ChIP) assay}

Lung cancer cells were infected with the indicated lentivirus, and ChIP assays were performed according to the manufacturer's instructions (Abcam, Cambridge, MA, USA). Briefly, cells were washed in PBS and cross-linked with $1 \%$ formaldehyde at room temperature for $10 \mathrm{~min}$. The cells were lysed and sonicated to shear DNA. Supernatant was obtained by centrifugation for $10 \mathrm{~min}$ at $8000 \times$ g. Samples were added to protein A/G beads and incubated overnight with anti-GSK3 $\beta$ or control IgG. After washing, DNA was eluted from the immunecomplex and quantitatively measured by SYBR green real-time quantitative PCR. The qPCR primers for the GSK3 $\beta$ promoter were $5^{\prime}$ - GGCTTCCCAGCGTCACTTTC $-3^{\prime}$ and $5^{\prime}$ - ACTCGTCCTCCACCTCCTTC $-3^{\prime}$.

\section{In vivo tumor xenograft studies}

The animal study was performed in accordance with the instructions of the Experimental Animal Care Commission of the Shanghai Jiao Tong University Affiliated Sixth People's Hospital. Four-to-five-week-old BALB/c nude mice (SLAC Animal, Shanghai, China; $n=12$ ) were housed in specific pathogen-free (SPF) conditions on a 12-h light/dark cycle with free access to food and water. NCI-H1975 cells infected with HOXA4-expressing or vector virus were injected subcutaneously into nude mice $\left(4 \times 10^{6}\right.$ cells in $\left.100 \mu \mathrm{l} \mathrm{PBS}\right)$. Tumor size was measured every 3 days for 33 days. At 33 days after cell transplantation, the mice were killed. The xenograft tumors were resected and weighed. The tumors were subjected to western blotting and immunohistochemistry (IHC) staining.

\section{IHC analysis}

The tumors were fixed in 10\% formalin, embedded in paraffin, and cut into $5-\mu \mathrm{m}$-thick sections. After routine deparaffinization, rehydration and inactivation of endogenous peroxidases, the sections were immersed in 0.01 $\mathrm{M}$ citrate buffer ( $\mathrm{pH}$ 6.0) and treated with microwaves for 15 min. Following incubation with $10 \%$ normal goat serum for $30 \mathrm{~min}$, the sections were incubated with a Ki67 antibody (Abcam) overnight at $4{ }^{\circ} \mathrm{C}$ and then with an HRP-conjugated secondary antibody for $1 \mathrm{~h}$ at room 
temperature. The sections were stained with the 3,3-diaminobenzidine (DAB) solution (Vector Laboratories, Burlingame, CA, USA) and counterstained with hematoxylin.

\section{Statistical analysis}

All data were analyzed using GraphPad Prism (San Diego, CA, USA). Student's $t$-test was used to compare differences between two groups, while one-way ANOVA was used to compare differences among more than two groups. The correlation between HOXA4 expression and clinical pathological features was assessed using Fisher's exact test. Kaplan-Meier analysis and the log-rank test were performed to compare the overall survival of patients with low or high tumor HOXA4 expression. $P<$ 0.05 was considered statistically significant.

\section{Acknowledgements}

This study was supported by the Shanghai Pudong New Area Science and Technology Development Fund (PKJ2014-Y06).

\section{Author details}

'Department of Thoracic-cardiovascular Surgery, Shanghai Jiao Tong University Affiliated Sixth People's Hospital, Shanghai, China. ${ }^{2}$ Department of Laboratory Medicine, Shanghai Jiao Tong University Affiliated Sixth People's Hospital, Shanghai, China. ${ }^{3}$ Department of Pathology, Shanghai Jiao Tong University Affiliated Sixth People's Hospital, Shanghai, China

\section{Conflict of interest}

The authors declare that they have no conflict of interest.

\section{Publisher's note}

Springer Nature remains neutral with regard to jurisdictional claims in published maps and institutional affiliations.

Received: 14 November 2017 Revised: 1 March 2018 Accepted: 13 March 2018

Published online: 27 April 2018

\section{References}

1. Torre, L. A. et al. Global cancer statistics, 2012. CA Cancer J. Clin. 65, 87-108 (2015).

2. Longo-Sorbello, G. S., Chen, B., Budak-Alpdogan, T. \& Bertino, J. R. Role of pemetrexed in non-small cell lung cancer. Cancer Invest. 25, 59-66 (2007).

3. Hirsch, F. R. et al. Epidermal growth factor receptor in non-small-cell lung carcinomas: correlation between gene copy number and protein expression and impact on prognosis. J. Clin. Oncol. 21, 3798-3807 (2003).

4. Nicholson, R. I., Gee, J. M. \& Harper, M. E. EGFR and cancer prognosis. Eur. J. Cancer 37(Suppl 4), S9-S15 (2001).

5. Brambilla, E., Moro, D., Gazzeri, S. \& Brambilla, C. Alterations of expression of Rb, p16(INK4A) and cyclin D1 in non-small cell lung carcinoma and their clinical significance. J. Pathol. 188, 351-360 (1999).

6. Stewart, D. J. Wnt signaling pathway in non-small cell lung cancer. J. Natl Cancer Inst. 106, djt356 (2014).

7. Haber, D. et al. Molecular targeted therapy of lung cancer: EGFR mutations and response to EGFR inhibitors. Cold Spring Harb Symp Quant Biol 70, 419-426 (2005).

8. Kumarakulasinghe, N. B., van Zanwijk, N. \& Soo, R. A. Molecular targeted therapy in the treatment of advanced stage non-small cell lung cancer (NSCLC). Respirology 20, 370-378 (2015).

9. Krumlauf, R. Hox genes in vertebrate development. Cell 78, 191-201 (1994).
10. Akam, M. Hox genes: from master genes to micromanagers. Curr. Biol. 8, R676-R678 (1998)

11. Galliot, B. et al. The mouse Hox-1.4 gene: primary structure, evidence for promoter activity and expression during development. Development 107, 343-359 (1989).

12. Behringer, R. R. et al. Sequences $5^{\prime}$ of the homeobox of the Hox-1.4 gene direct tissue-specific expression of lacZ during mouse development. Development 117, 823-833 (1993).

13. Packer, A. I., Crotty, D. A., Elwell, V. A. \& Wolgemuth, D. J. Expression of the murine Hoxa4 gene requires both autoregulation and a conserved retinoic acid response element. Development 125, 1991-1998 (1998).

14. Packer, A. I., Mailutha, K. G., Ambrozewicz, L. A. \& Wolgemuth, D. J. Regulation of the Hoxa4 and Hoxa5 genes in the embryonic mouse lung by retinoic acid and TGFbeta1: implications for lung development and patterning. Dev. Dyn. 217, 62-74 (2000)

15. Bhatlekar, S. et al. Identification of a developmental gene expression signature, including HOX genes, for the normal human colonic crypt stem cell niche: overexpression of the signature parallels stem cell overpopulation during colon tumorigenesis. Stem. Cells Dev. 23, 167-179 (2014).

16. Yamashita, T. et al. Suppression of invasive characteristics by antisense introduction of overexpressed HOX genes in ovarian cancer cells. Int. J. Oncol. $\mathbf{2 8}$ 931-938 (2006).

17. Klausen, C., Leung, P. C. \& Auersperg, N. Cell motility and spreading are suppressed by HOXA4 in ovarian cancer cells: possible involvement of beta1 integrin. Mol. Cancer Res. 7, 1425-1437 (2009).

18. Plowright, L., Harrington, K., Pandha, H. \& Morgan, R. HOX transcription factors are potential therapeutic targets in non-small-cell lung cancer (targeting $\mathrm{HOX}$ genes in lung cancer). Br. J. Cancer 100, 470 (2009).

19. Abe, M. et al. Disordered expression of HOX genes in human non-small cell lung cancer. Oncol. Rep. 15, 797-802 (2006).

20. Polakis, P. Wnt signaling in cancer. Cold Spring Harb. Perspect. Biol. 4, a008052 (2012).

21. Li, C. et al. Evidence of the cross talk between Wnt and Notch signaling pathways in non-small-cell lung cancer (NSCLC): Notch3-siRNA weakens the effect of LiCl on the cell cycle of NSCLC cell lines. J. Cancer Res. Clin. Oncol. 137 771-778 (2011).

22. Stewart D. J. Wht signaling pathway in non-small cell lung cancer. J. Natl Cancer Inst. 106, djt356 (2014).

23. Ordóñez-Morán, P., Dafflon, C., Imajo, M., Nishida, E. \& Huelsken, J. HOXA5 counteracts stem cell traits by inhibiting Wnt signaling in colorectal cancer. Cancer Cell. 28, 815-829 (2015).

24. Ferrell, C. M. et al. Activation of stem-cell specific genes by HOXA9 and HOXA10 homeodomain proteins in CD34+ human cord blood cells. Stem Cells 23, 644-655 (2005)

25. Kase, $\mathrm{S}$. et al. Expression of E-cadherin and $\beta$-catenin in human nonsmall cell lung cancer and the clinical significance. Clin. Cancer Res $\mathbf{6}$, 4789-4796 (2000)

26. Gautschi, O., Ratschiller, D., Gugger, M., Betticher, D. C. \& Heighway, J. Cyclin D1 in non-small cell lung cancer: a key driver of malignant transformation. Lung Cancer 55, 1-14 (2007).

27. Betticher, D. C. et al. Prognostic significance of CCND1 (cyclin D1) overexpression in primary resected non-small-cell lung cancer. Br. J. Cancer 73, 294 (1996).

28. Chen, Z. et al. miRNA-145 inhibits non-small cell lung cancer cell proliferation by targeting c-Myc. J. Exp. Clin. Cancer Res. 29, 151 (2010).

29. Krysan, K., Dalwadi, H., Sharma, S., Põld, M. \& Dubinett, S. Cyclooxygenase 2dependent expression of survivin is critical for apoptosis resistance in nonsmall cell lung cancer. Cancer Res. 64, 6359-6362 (2004).

30. Ding, Q. et al. Myeloid cell leukemia-1 inversely correlates with glycogen synthase kinase-3 $\beta$ activity and associates with poor prognosis in human breast cancer. Cancer Res. 67, 4564-4571 (2007).

31. $\mathrm{Ma}, \mathrm{C}$. et al. The role of glycogen synthase kinase $3 \beta$ in the transformation of epidermal cells. Cancer Res. 67, 7756-7764 (2007).

32. Farago, M. et al. Kinase-inactive glycogen synthase kinase $3 \beta$ promotes Wnt signaling and mammary tumorigenesis. Cancer Res. 65, 5792-5801 (2005).

33. Wang, Y., Lam, K. S. \& Xu, A. Adiponectin as a negative regulator in obesityrelated mammary carcinogenesis. Cell Res. 17, 280-282 (2007).

34. Zheng, H., Saito, H., Masuda, S., Yang, X. \& Takano, Y. Phosphorylated GSK3ßser9 and EGFR are good prognostic factors for lung carcinomas. Anticancer Res. 27, 3561-3569 (2007) 
35. Zhou, B. P. \& Hung, M.C. Wnt, hedgehog, and snail: sister pathways that control by GSK-3beta and beta-Trcp in the regulation of metastasis. Cell Cycle 4, 772-776 (2005).

36. Yook, J. I. et al. A Wnt-Axin2-GSK3ß cascade regulates Snail1 activity in breast cancer cells. Nat. Cell. Biol. 8, 1398-1406 (2006).

37. Shakoori, A. et al. Deregulated GSK3 $\beta$ activity in colorectal cancer: its association with tumor cell survival and proliferation. Biochem. Biophys. Res. Commun. 334, 1365-1373 (2005).

38. Zeng, J. et al. GSK3 $\beta$ overexpression indicates poor prognosis and its inhibition reduces cell proliferation and survival of non-small cell lung cancer cells. PLoS ONE 9, e91231 (2014).

39. Ougolkov, A. V., Fernandez-Zapico, M. E., Savoy, D. N., Urrutia, R. A. \& Billadeau, D. D. Glycogen synthase kinase-3beta participates in nuclear factor kappaBmediated gene transcription and cell survival in pancreatic cancer cells. Cancer Res. 65, 2076-2081 (2005).
40. Naito, S. et al. Glycogen synthase kinase-3ß: a prognostic marker and a potential therapeutic target in human bladder cancer. Clin. Cancer Res. 16 5124-5132 (2010)

41. Cao, Q., Lu, X. \& Feng, Y.-J. Glycogen synthase kinase-3 $\beta$ positively regulates the proliferation of human ovarian cancer cells. Cell Res. 16 671-677 (2006).

42. Livak, K. J. \& Schmittgen, T. D. Analysis of relative gene expression data using real-time quantitative PCR and the 2(-Delta Delta C(T)) Method. Methods 25 402-408 (2001).

43. Subramanian, A. et al. Gene set enrichment analysis: a knowledge-based approach for interpreting genome-wide expression profiles. Proc. Natl Acad. Sci. USA 102, 15545-15550 (2005).

44. Kim, H.S. et al. C-terminal fragments of amyloid precursor protein exert neurotoxicity by inducing glycogen synthase kinase-3 $\beta$ expression. FASEB $\mathrm{J}$. 17, 1951-1953 (2003) 